\title{
A novel recombinant immunocasp-6 fusion gene specifically and efficiently suppresses HER2-overexpressing osteosarcoma
}

\author{
BEN-GEN ZHOU ${ }^{1,2^{*}}$, MEI-YUAN LIU ${ }^{3 *}$, XIU-CHUN QIU ${ }^{4}$, YAN-MING XU ${ }^{5}$, \\ QING-YU FAN ${ }^{4}$, AN-GANG YANG $^{6}$, YU ZHANG $^{2}$ and HONG XIA ${ }^{2}$ \\ ${ }^{1}$ Graduate School of Southern Medical University; ${ }^{2}$ Department of Orthopaedics, Guangzhou General Hospital \\ of Guangzhou Military Command; ${ }^{3}$ Affiliated Tumor Hospital of Guangzhou Medical College, Guangzhou, \\ Guangdong; ${ }^{4}$ Department of Orthopaedic Surgery, Tangdu Hospital, Fourth Military Medical University; \\ ${ }^{5}$ State Key Laboratory of Cancer Biology, Department of Biochemistry and Molecular Biology, Fourth Military \\ Medical University; ${ }^{6}$ Department of Immunology, Fourth Military Medical University, Shaanxi, Xi'an, P.R. China
}

Received August 29, 2012; Accepted September 25, 2012

DOI: 10.3892/or.2012.2122

\begin{abstract}
Osteosarcoma is the most common primary malignant tumor of bone for adolescent or children. The poor prognosis of patients, due to its remote metastasis, has led to the exploration of more effective and less toxic treatments. Immunotherapy is a promising strategy for the treatment of human epidermal growth factor receptor 2 (HER2)-overexpressing tumors. Herein, we describe experiments conducted with a fusion gene, immunocasp-6, which was generated by fusing a HER2-specific single-chain Ab, a single-chain Pseudomonas exotoxin A and an active caspase-6 which can directly cleave lamin A leading to nucleus damage inducing programmed cell death. We demonstrated that immunocasp- 6 can specifically and efficiently recognize and induce apoptosis in HER2-overexpressing osteosarcoma cells in vitro. The immunocasp- 6 was transferred into BALB/c athymic mice bearing human osteosarcoma by i.m. injection of liposome-encapsulated pCMV-immunocap-6. Expression of immunocasp-6 not only strongly inhibited tumor growth and significantly prolonged animal survival, but also greatly prevented tumor metastasis. Our data showed that the immunocasp-6 can specifically recognize HER2-overexpressing osteosarcoma cells, can also promptly attack their nucleus and induce apoptotic death, suggesting the potential of this strategy for the treatment of human HER2-overexpressing tumors.
\end{abstract}

Correspondence to: Professor Hong Xia, Department of Orthopaedics, Guangzhou General Hospital of Guangzhou Military Command, 111 Liuhua Road, Guangzhou, Guangdong 510010, P.R. China

E-mail: gzxiahong2@126.com

*Contributed equally

Key words: osteosarcoma, gene therapy, human epidermal growth factor receptor-2, caspase-6, apoptosis

\section{Introduction}

The human epidermal growth factor receptor-2 (HER2), a member of the epithelial growth factor receptor family, transduces cell signaling and plays key roles in cell differentiation, adhesion, and motility (1). Sufficient evidence has suggested that patients with HER2-overexpressing tumors exhibit a reduced response to conventional treatments (2). The HER2 protein is reportedly overexpressed in several human malignant tumor, including human breast and ovarian cancer (3), salivary gland adenocarcinoma (4), gastric cancer (5) and osteosarcoma (6-9). Since it is overexpressed in tumor cells but is not detected in normal cells, HER2 is an ideal target molecular for cancer gene therapy to exploit differences at the molecular level between normal and malignant cells (10).

Caspases are vital elements in transferring apoptotic signals and executing apoptosis in mammalian cells (11). Caspase- 6 is one of effective caspases during the cell apoptotic program (12). Activation of caspase-6 induces apoptosis by cleaving lamin A and other substrates (13). Unlike its wildtype zymogen counterpart, active caspase- 6 constructed with subunits in reverse order, is capable of autocatalytic processing in vitro independent of apoptotic signals, and can induce apoptosis of tumor cells, which thereby makes it an attractive candidate for gene therapy (14).

As a well-recognized $\mathrm{Ab}, \mathrm{e} 23 \mathrm{sFv}$, derived from a mouse mAb against human HER2, has been confirmed to bind the extracellular domain of HER2 protein with high affinity and to be internalized by endocytosis $(15,16)$. The highly specific antibody to antigen suggests that we can construct a fusion gene, immunocasp-6, consisting of NH2-terminal leader sequence to promote secretion of the recombinant immunocasp- 6 fused with an anti-HER2 single-chain Ab, the translocation domain (domain II) of Pseudomonas exotoxin A (PEA) and an active caspase-6, to specifically and efficiently suppress the HER2 overexpressing tumors. PEA is a single-chain toxin consisting of three major domains (I, II and III) responsible for binding of the molecule to target cells, translocation of the molecule to the cytosol, and the induction of cell death, respectively (17). 
caspase-6

prodomain large subunit small subunit

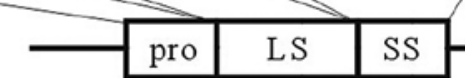

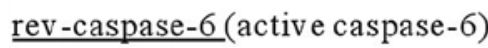
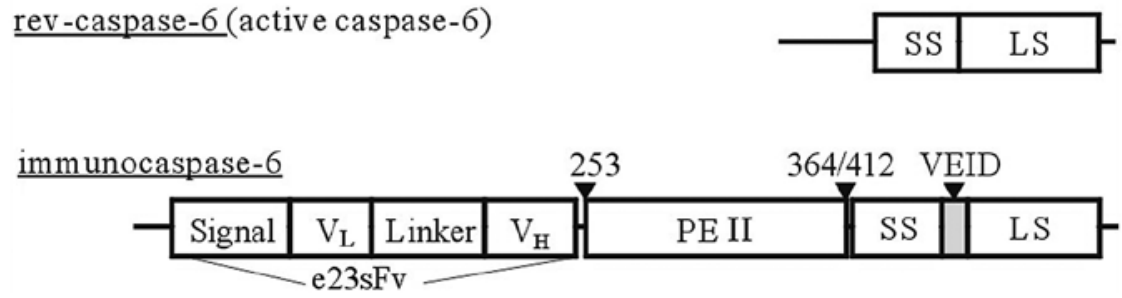

Figure 1. Schematic diagram of immunocasp- 6 constructs. Immunocasp- 6 construct was generated by fusing the e $23 \mathrm{sFv}$ gene in frame with the 5 ' end of the fusion genes encoding the PEA domain II (amino acids 253-412) and active caspase-6.

Domain II of PEA has been reported to efficiently transfer the cellular toxicity domain to the cytoplasm (18-20). By replacing the cellular toxicity domain of PEA with active caspase-6, we sought to translocate the caspase into tumor cells in which it would induce tumor cell apoptosis. Even though this novel immunocasp-6 has been proven to be effective in inducing apoptosis in the HER2-overexpressing human breast tumor cell line, SKBR-3, their effects on human osteosarcoma is still unclear. Thus the purpose of the present study is to extend our immunocasp-6 strategy to the treatment of osteosarcoma in vitro as well as in vivo and to verify that the immunocasp- 6 can specifically and efficiently suppress the HER2-overexpressing tumors.

\section{Materials and methods}

Plasmid and DNA construct. Recombinant immunocasp-6 was generated by sequential fusion of the genes of a signal peptide (Met-Lys-His-Leu-Trp-Phe- Phe-Leu-Leu-Leu-Val-Ala-AlaPro-Arg-Trp-Val-Leu-Ser-) consisting of a single chain HER2 antibody (e23sFv), a Pseudomonas exotoxin A (PEA) translocation domain (from aa 253 to 412) and an active caspase-6 (Fig. 1). The immunocasp-6 was cloned into a pCMV plasmid, namely pCMV-immunocasp-6.

Cell culture and transfection. Human osteosarcoma cell line SOSP-9607-E10, with relatively high metastatic potential, was derived from a 17-year-old male patient who had been diagnosed of tibial osteosarcoma and underwent osteotomy and established from these cells by continuous in vitro cultivation for over 120 transfer generations in one year. SOSP-9607-E10 cells were maintained in DMEM or RPMI-1640 (Invitrogen) supplemented with $10 \%$ fetal bovine serum (FBS) and $4 \mathrm{mmol} / 1$ L-glutamine. At $24 \mathrm{~h}$ before transfection, cells were seeded in 12 or 96 -well plates at $1 \times 10^{5}$ or $5 \times 10^{3}$ cells per well. pCMVimmunocasp- 6 or pCMV vector alone, $1 \mu \mathrm{g}$, encapsulated by $2 \mu 1$ Lipofectamine 2000 (Invitrogen) were mixed, incubated for $20 \mathrm{~min}$ at room temperature to form DNA-liposome mixture. Then the mixture was administered to cells and incubated in a humidified incubator at $37^{\circ} \mathrm{C}$ with $5 \% \mathrm{CO}_{2}$ for $6 \mathrm{~h}$, then the medium was removed and cells were resuspended in complete medium.

Cell viability assay. Viability of the transiently transfected cells was tested by using the 3-(4,5-dimethylthiazol-2-yl)- 2,5-diphenyltetrazo-lium bromide (MTT) assay. Briefly, after the cells adhered to the low lamber of 96-well plates, the cells were divided randomly into three groups, namely the mock group, the control group and the immunocasp- 6 group, and transfected with pCMV-immunocasp-6 or pCMV vector. Thereafter, the cells were cultured in 96-well plates for 12 to $96 \mathrm{~h}$, then incubated with $20 \mu \mathrm{l}$ of $1.5 \mathrm{mg} / \mathrm{ml}$ MTT for $4 \mathrm{~h}$. After that, the cells were treated with $150 \mu 1$ DMSO. OD at A490 nm were determined using the Sunrise microplate reader (Tecan). Each assay was performed in triplicate on at least three independent occasions.

Flow cytometry assay for detection of apoptosis. SOSP9607-E10 cells were seeded at a density of $1 \times 10^{5}$ cells per well on slides in 12-well Costar transwell plates, and transfected with pCMV-immunocasp-6 or pCMV vector when cell number was at a density of $3 \times 10^{5}$ cells per well. After $48 \mathrm{~h}$ of transfection, cells in the lower chamber were collected, stained with Annexin V-FITC/PI following standard procedures and finally analyzed by FCM.

Immunofluorescence. After transfection SOSP-9607-E10 cells were incubated in complete medium for an additional $48 \mathrm{~h}$. Then, the cells in the lower chamber were fixed in paraformaldehyde solution (4\% in phosphate buffered saline (PBS), $\mathrm{pH}$ 7.4), permeabilized with PBS containing 0.1\% Triton X-100, and blocked with $2 \%$ normal rabbit serum. Then the cells were stained with antibodies recognizing caspase-6 (C20, 1:200; Santa Cruz Biotechnologies) as the primary antibodies, with biotin-linked anti-goat IgG (1:100; Santa Cruz Biotechnologies) and FITC-linked anti-rabbit IgG (1:100; Sigma) as the secondary antibodies. The staining was examined using a fluorescence microscope (Japanese Olympus Co.).

Electronic microscopy assay. SOSP-9607-E10 cells were harvested $48 \mathrm{~h}$ after transfection, and then fixed in $2.5 \%$ glutaraldehyde, dehydrated and embedded to observe morphologic change with a transmission electron microscope.

Immunohistochemistry assay. The transfected cells were cultured on coverslips as mention above, and then fixed with a freshly prepared paraformaldehyde solution for $30 \mathrm{~min}$ at room temperature, and permeabilized with $0.1 \%$ Triton $\mathrm{X}-100$ for 15 min on ice. 
A

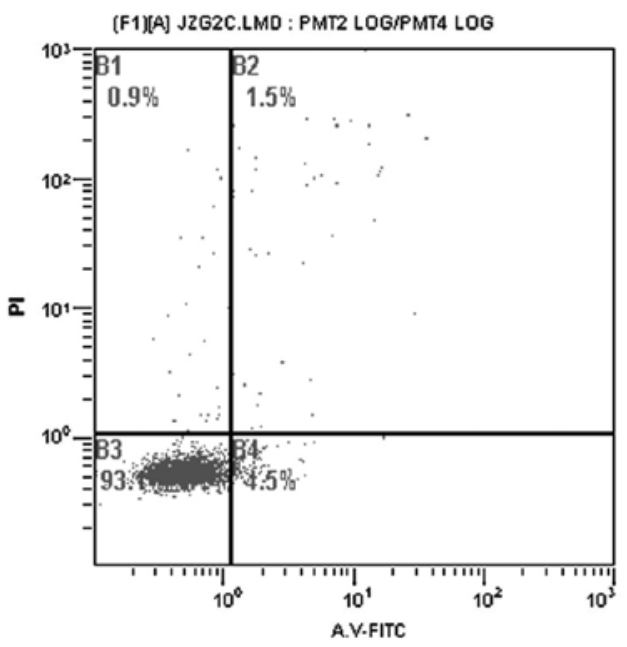

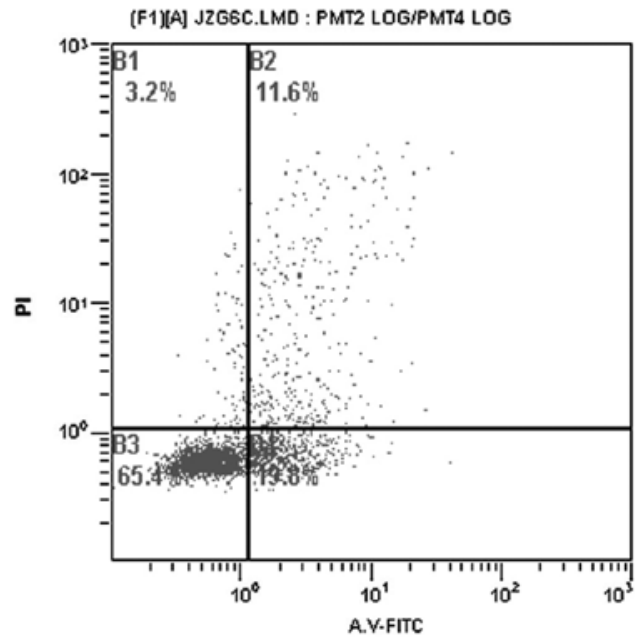

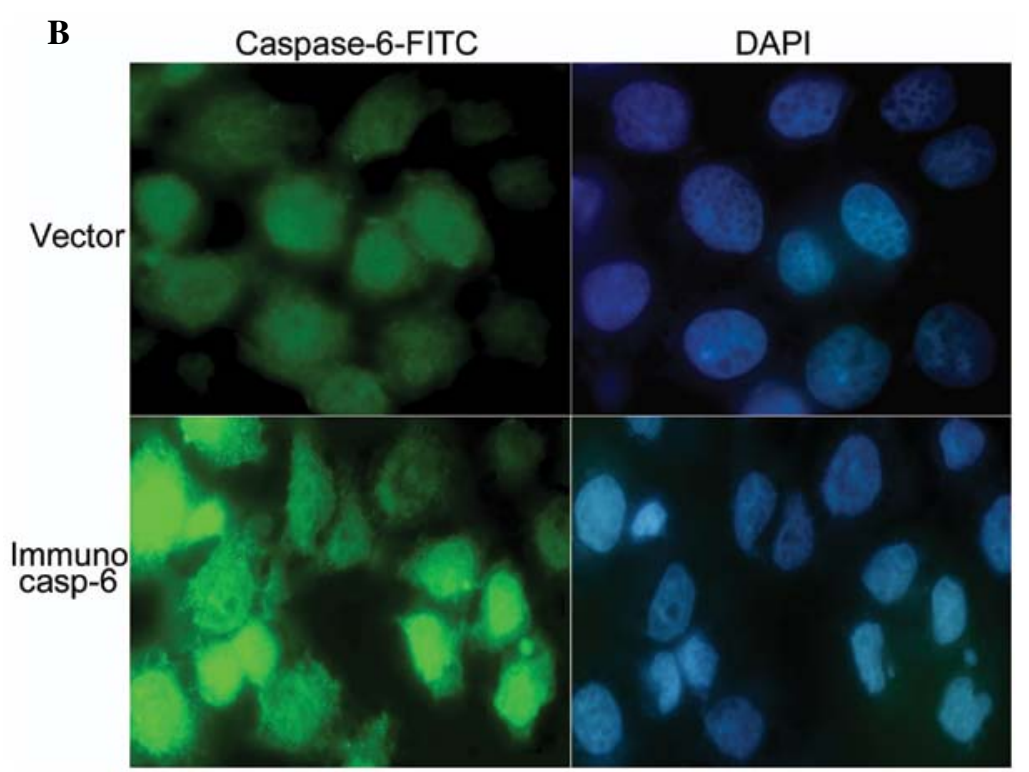

C

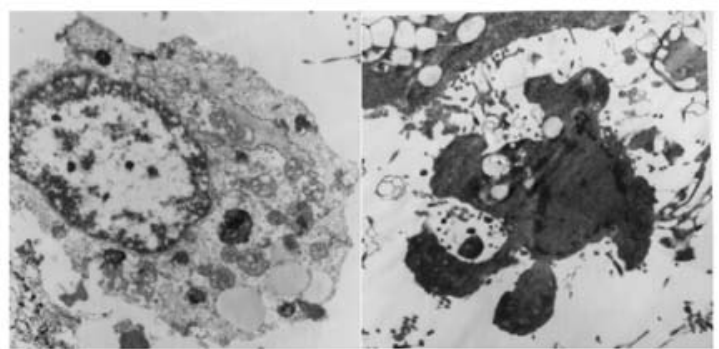

D

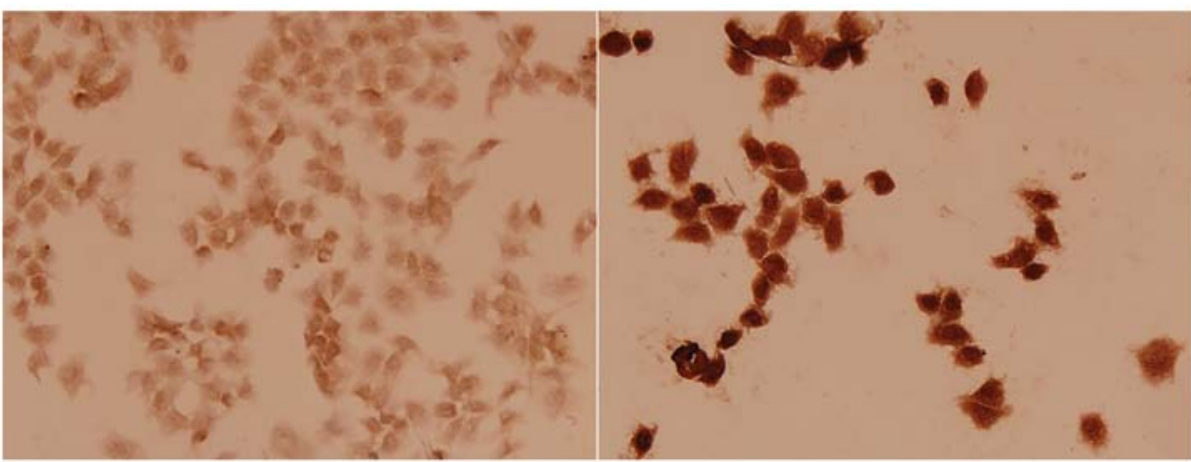

Figure 2. Immunocasp-6 induced apoptosis in HER2-overexpressing osteosarcoma cells. SOSP-9607-E10 cells were transiently transfected with immunocasp-6, and cell viability were tested using Annexin V-FITC staining (A). Morphological change of transfected cells were tested by immunofluorescence assay (B), and electronic microscopy (C). Immunohistology staining was performed on the transfected SOSP-9607-E10 cells with anti-caspase-6 antibody (D). The left part of (A-D) is the result of cells in the vector group while the right part is the result of cells in the immunocasp- 6 group.

Xenograft tumors and muscle tissues were fixed in paraformaldehyde solution and embedded in paraffin after treatment, paraffin-embedded tissue sections were dewaxed, hydrated, and incubated in $0.3 \%$ methanol- $\mathrm{H}_{2} \mathrm{O}_{2}$ for 20 min to remove endogenous peroxidase. Next, they were dried and blocked for $1 \mathrm{~h}$ with the appropriate serum in a humidified chamber. Primary antibody was added overnight at $4^{\circ} \mathrm{C}$.
The samples were probed with primary antibody-recognizing caspase-6 (C20, 1:200; Santa Cruz Biotechnologies), followed by biotin-linked antirabbit IgG (1:100; Santa Cruz Biotechnology) as the secondary antibody and then processed with the Vectastain Elite ABC kit per the manufacturer's instructions prior to digital photography under an Olympus Eclipse E600 microscope with a Spot RT slider camera and imaging software. 
TUNEL staining. TUNEL staining was performed on paraffin sections, using the TdT-FragEL ${ }^{\mathrm{TM}}$ DNA Fragmentation Detection kit (Calbiochem) in accordance with the manufacture's instructions. Hematoxylin was used to counterstain the sections.

Antitumor activity of immunocasp-6 in vivo. Six- to eightweek-old BALB/c athymic mice were purchased from National Rodent Laboratory Animal Resources, Shanghai Branch (Shanghai, China), and were cared and used in compliance with institutional guidelines. The mice were inoculated s.c. with $2 \times 10^{6}$ SOSP-9607-E10 cells. Tumors were allowed to grow until they reached a diameter of 5-7 $\mathrm{mm}$ (day 0 ). The mice were then randomly divided into different two groups, namely the immunocasp- 6 group and vector group.

The mice bearing SOSP-9607-E10 tumors were subjected to liposome-mediated immunocasp- 6 or vector treatments. pCMV-immunocasp- 6 or pCMV vector alone, $10 \mu \mathrm{g}$, encapsulated by $20 \mu 1$ Lipofectamine 2000 was administered i.m. to mice. Nine mice were utilized for each treatment, each mouse was administered every 3 days for a total of five times. The volume of the tumor, the body weight of the mice and the net weight of the tumor were observed and analyzed by statistics.

Assessment of immunocasp- 6 effects on human osteosarcoma lung metastasis. Eighteen athymic, six- to eight-week-old $\mathrm{Balb} / \mathrm{c}$ mice were inoculated with $2 \times 10^{5}$ SOSP-9607-E10 cells into thighbone marrow. The mice were then divided randomly into two groups, nine mice in each group, for i.m. liposomemediated immunocasp- 6 or vector treatments as indicated above. The treatment was performed once every 3 days for two weeks, then once a week thereafter for five weeks. The number of the neonatal tumors in lungs were counted, mouse survival times were recorded and the neonatal mass was tested by $\mathrm{H} \& \mathrm{E}$ staining.

Statistical analyses. The data are expressed as the mean $\pm \mathrm{SD}$. Statistical analyses were performed with the SPSS13.0 software package for Windows (SPSS, Chicago, IL). Cell viability assay were analyzed by the analysis of covariance (ANCOVA, dunnett T3) method. The volume of the tumor, the body weight of the mice and the net weight of the tumor were analyzed using independent-samples t-test (the data of the volume of the tumor and the body weight of the mice were obtained from the data before treatment subtracted by the data after treatment). The survival rates were analyzed using the KaplanMeier method, and comparisons among treatment groups were obtained using the log-rank test. Statistical significance was based on a value of $\mathrm{P}<0.05$.

\section{Results}

Immunocasp- 6 effectively and specifically suppresses the growth of SOSP-9607-E10 cells in vitro. Active caspase-6 is expressed and secreted from the transfected cells, binds to HER2-overexpressing breast tumor cells, internalizes, undergoes autoprocessing between PEA Arg279 and Gly280, and induces cell apoptosis (21). To investigate whether the same cytotoxic effect could be achieved in osteosarcoma cells, we first tested the cell viability of the transiently transfected 
A

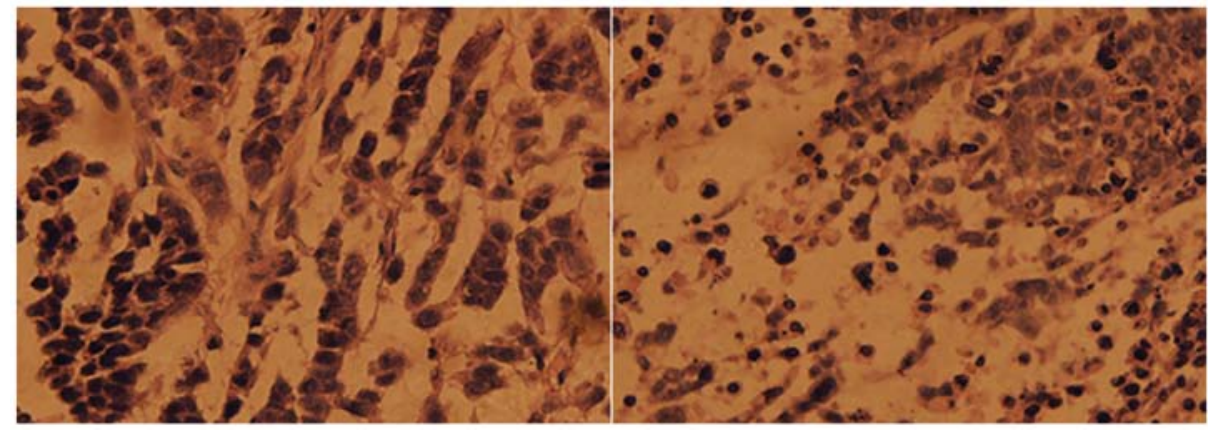

B

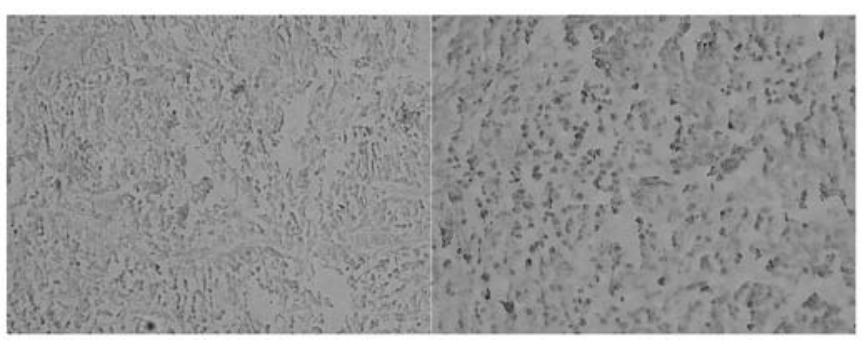

D

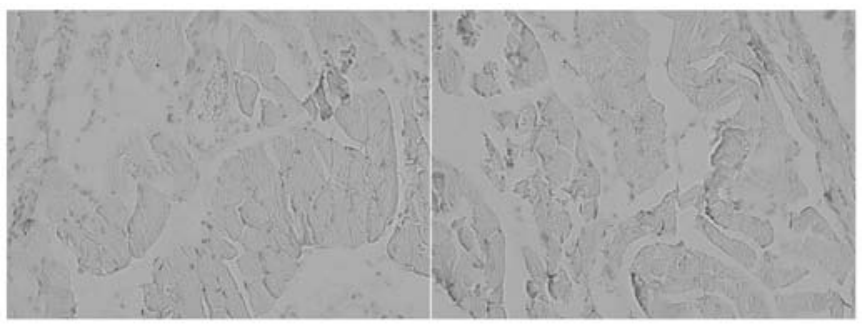

Figure 3. Immunocasp-6 suppressed the growth of osteosarcoma in vivo. The SOSP-9607-E10 nude mice were randomly divided into two groups. They received i.m. injections of $10 \mu \mathrm{g}$ of lipofectamine-encapsulated pCMVimmunocasp -6 or pCMV vector plasmid in the right posterior limb every 3 days for a total of five times. The body volume of the tumor, body weight of the mice and net weight of the tumor were observed and analyzed by statistics (Table II). The tumor tissues were collected and subjected to H\&E staining (A), TUNEL staining (B). The tumor and muscle tissues were tested by immunohistochemistry staining with caspase- 6 antibody $(\mathrm{C}$ and $\mathrm{D})$. The left part of (A-D) is the result of tissue in the vector group, while the right part is the result of tissue in the immunocasp- 6 group.

cells by MTT assay, Table I shows that transient expression of the immunocasp-6 led to an apparent delay in cell viability. In other words, tumors cells in the immunocasp- 6 treatment group have weaker viability than those in the mock or control group ( $\mathrm{P}=0.013, \mathrm{P}=0.007$, respectively), while there is no significantly difference between the mock group and control group $(\mathrm{P}=0.989)$.

The Annexin V-FITC staining $48 \mathrm{~h}$ after the transfection revealed that the percentages of apoptotic cells in the immunocasp-6 group were $31.4 \%$, while only $6 \%$ in the vector group (Fig. 2A). When tumor cell growth was stunted, the morphological change of transfected cells were tested.Immunofluorescence test discovered that transiently transfected cells had enriched or chipped nuclear (Fig. 2B). Transmission electron microscopy presented typical apoptotic changes in cells, including chromatin condensation and its margination at the nuclear periphery, cellular shrinkage and blebbing, and formation of so-called
C

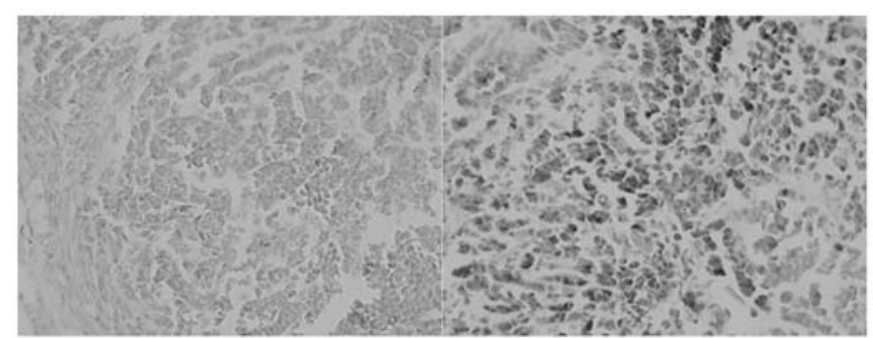

apoptotic bodies (Fig. 2C). Furthermore, immunohistochemistry staining with anti-caspase- 6 antibody revealed that most of the cells were caspase- 6 positive, suggesting that caspase- 6 was effective in inducing tumor cell apoptosis.

Immunocasp-6 transduction-induced HER2-overexpressing osteosarcoma cell death in subcutaneously transplanted nude mice. As showed in Table II, the growth of the tumor in the liposome-mediated pCMV-immunocasp- 6 treatment group was significantly slower than that of the $\mathrm{pCMV}$ vector group $(\mathrm{P}=0.001)$, the body weight of the mice in the immunocasp-6 group was significantly heavier than that of the vector group $(\mathrm{P}=0.0002)$, and the net weight of the tumor in the immunocasp- 6 group was significantly less than that of the vector group $(\mathrm{P}=0.0006)$. Then the osteosarcoma tissues were collected and H\&E (hematoxylin and eosin) stain was performed. The staining discovered tumor tissues were in poor condition in which most of tumor tissue was dead (Fig. 3A). Furthermore, terminal deoxynucleotidyl transferase-mediated dUTP nick-end-labeling (TUNEL) staining, demonstrated that most of the tumor tissue in the immunocasp-6 group were in the state of apoptosis (Fig. 3B). Thereafter, immunohistochemistry analysis confirmed the presence of caspase- 6 in tumor tissues in the immunocasp- 6 group, but not in those treated with vector (Fig. 3C) and muscle tissues in either immunocasp- 6 group or vector group (Fig. 3D). These findings suggest that immunocasp-6 can specifically and efficiently induce tumor tissue apoptosis.

Inhibitory effect of immunocasp-6 on lung metastasis of HER2overexpressing osteosarcoma in vivo. The neonatal mass were diagnosed as osteosarcoma by H\&E staining (data not shown). As shown in Fig. 4A, the number of neonatal tumors in lungs in the immunocasp- 6 group was significantly less than that in the vector group. Furthermore, the mice in the immunocasp- 6 group survived longer than that in the vector group (Fig. 4B). SOSP-9607-E10 tumors were more likely to metastasize to the 
Table II. Comparison between control and treatment group of the tumor volume, tumor net weight and mouse body weight $($ mean $\pm \mathrm{SD})$.

\begin{tabular}{lcccc}
\hline Group & $\mathrm{n}$ & Tumor volume $\left(\mathrm{mm}^{3}\right)$ & Tumor net weight $(\mathrm{g})$ & Mouse body weight $(\mathrm{g})$ \\
\hline Control & 9 & $975.09 \pm 49.76$ & $1.08 \pm 0.16$ & $6.20 \pm 1.14$ \\
Treatment & 9 & $376.01 \pm 265.18^{\mathrm{a}}$ & $0.64 \pm 0.18^{\mathrm{b}}$ & $4.07 \pm 0.49^{\mathrm{c}}$ \\
\hline
\end{tabular}

${ }^{\mathrm{a}} \mathrm{P}=0.001,{ }^{\mathrm{b}} \mathrm{P}=0.0006,{ }^{\mathrm{c}} \mathrm{P}=0.0002$ (compared with the control group).

A

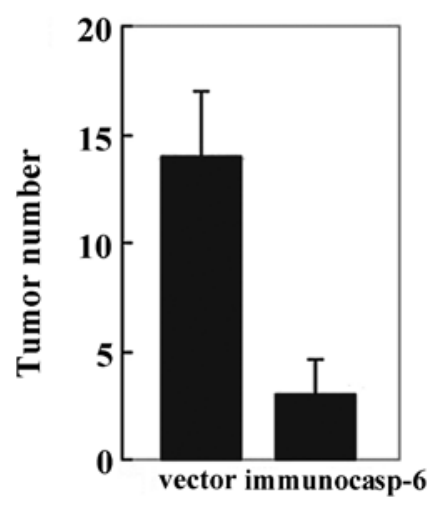

B

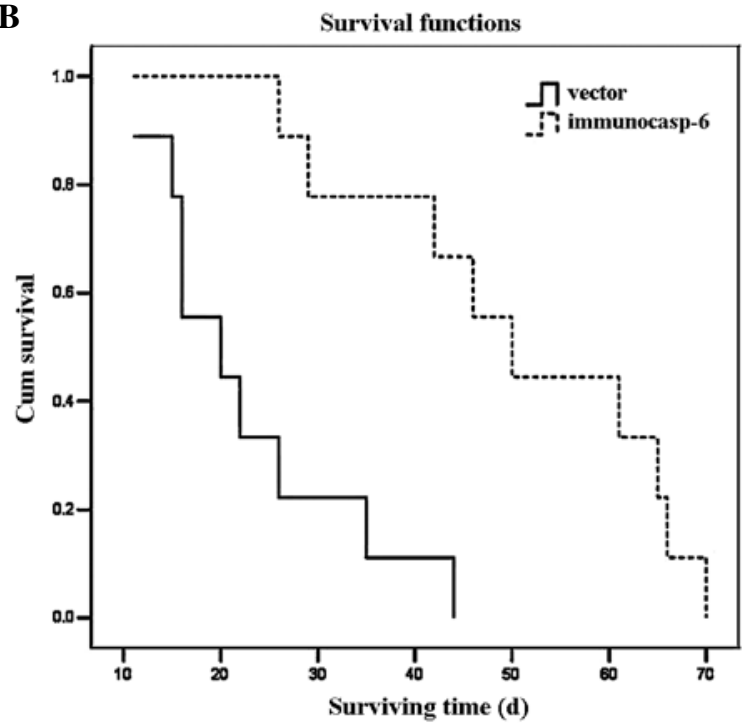

Figure 4. Immunocasp-6 treatment suppresses the metastasis of HER2overexpressing osteosarcoma in vivo. Tumor numbers in lung tissue were counted (A). Survival rates of immunocasp- 6 treated and control mice were evaluated (B).

lung in the absence of immunocasp- 6 treatment, suggesting that immunocasp- 6 can prevent or slow osteosarcoma metastasis.

\section{Discussion}

Many strategies of gene therapy have been designed to kill cancer cells, including their transduction with suicide genes or tumor suppressor genes or activation of the immune system against the tumor cells (22-25). Although many problems have impeded the practical use of gene-based therapy for human cancers, the search for safe, effective and tissue-specific gene therapies continues. In the past decade, we carried out a series of studies on antibody-directed and cell-mediated cancer immunotherapy by combining the specificity of antibodies and the potent cytotoxicity of pro-aoptotic proteins. Up to now, a number of pro-apoptotic effectors, including caspase-3 (26), caspase-6 (21), granzyme B (27), tBid (10) and apoptosis inducing factor (AIF) (28), have been used to construct immunoproapoptotic proteins and have been confirmed to be efficient in inducing targeted apoptosis both in vitro and in vivo. In the present study, we generated a novel immunocasp -6 gene construct by fusing a leader sequence, single-chain HER2 (e23sFv) Ab and the translocation domain of PEA to the active caspase- 6 and extended the strategy to HER2 overexpression osteosarcoma.

In the study, we verified that the HER2-targeted suppressing effect of the novel immunocap- 6 on osteosarcoma cells in vitro. Firstly, we transfected the fusion gene into SOSP9607-E10 cells and compared the vitality of these tumor cells. The effective destruction of SOSP-9607-E10 cells by the novel immunocasp- 6 was confirmed by both MTT assay and flow cytometry assay. These findings suggested that the immunocasp-6 can strongly inhibit the growth of SOSP-9607-E10 cells. Morphological examination including immunofluorescence assay and electron microscopy verified that SOSP-9607-E10 cells in the immunocasp- 6 group presented the typical characteristics of apoptosis, which suggested that immunocasp-6 might inhibit the growth of the tumor cells by inducing tumor cell apoptosis. In order to further explore the factors responsible for tumor cell apoptosis, the immunohistochemistry analysis were applied. The findings verified that caspase- 6 can lead to tumor cell apoptosis. Thus, it can be concluded that the novel immunocasp- 6 can specifically recognize the HER2-overexpressing osteosarcoma cells, induce tumor cell apoptosis and strongly inhibit the growth of tumor cells.

In order further to verify HER2-targeted suppressing effect of the novel immunocap -6 on the osteosarcoma cells in vivo, the mouse SOSP-9607-E10 tumor xenograft model was constructed. The mice in the immunocasp- 6 group showed better condition than that in the vector group, the growth of the tumor became slower $(\mathrm{P}=0.001)$, the weight of the nude mice was heavier $(\mathrm{P}=0.0006)$, the net weight of the tumor was lighter $(\mathrm{P}=0.0002)$, which suggested the immunocasp- 6 can suppress the growth of the tumors. Thereafter, H\&E staining and TUNEL staining revealed the tumor tissue treated by immunocasp- 6 was also in poor condition and presented the character of apoptosis, suggesting that immunocasp-6 suppressed the growth of the tumor by inducing tumor tissue apoptosis. Furthermore, immunohistochemistry analysis 
confirmed the presence of caspase- 6 in tumor tissues treated with immunocasp- 6 , while caspase- 6 were not found in those treated with pCMV vector and muscle tissues in either treatment group or control group. Thus, it can be concluded that the novel immunocasp- 6 can specifically recognize and efficiently suppress the growth of HER2-overexpressing osteosarcoma, without damaging the normal tissues.

Moreover, in order to verify if the immunocasp- 6 can prevent the metastasis of SOSP-9607-E10 tumors, we counted the number of neonatal tumors in lungs after mice died and recorded the survival time. The findings showed that the neonatal tumors in lungs in the immunocasp- 6 group were significantly less than that in the vector group. Furthermore, the mice in the immunocasp- 6 group survived longer than that in the vector group. SOSP-9607-E10 tumors were more likely to metastasize to the lung in the absence of immunocasp- 6 treatment suggesting that immunocasp- 6 prevents or slows osteosarcoma metastasis.

In summary, we described a novel immunocasp-6 therapeutic gene construct, which can kill HER2-overexpressing osteosarcoma specifically and efficiently; this novel immunocasp- 6 holds promise for the generation of a novel therapy for HER2-overexpressing tumors.

\section{Acknowledgements}

This study was supported by grants from the National Natural Science Foundation of China (No. 30330610).

\section{References}

1. Lupu R, Colomer R, Kannan B and Lippman ME: Characterization of a growth factor that binds exclusively to the erbB-2 receptor and induces cellular responses. Proc Natl Acad Sci USA 89: 2287-2291, 1992.

2. Chen JS, Lan K and Hung MC: Strategies to target HER2/neu overexpression for cancer therapy. Drug Resist Updat 6: 129-136, 2003.

3. Slamon DJ, Godolphin W, Jones LA, et al: Studies of the HER-2/ neu proto-oncogene in human breast and ovarian cancer. Science 244: 707-712, 1989

4. Semba K, Kamata N, Toyoshima K and Yamamoto T: A v-erbBrelated protooncogene, c-erbB-2, is distinct from the c-erbB-1/ epidermal growth factor-receptor gene and is amplified in a human salivary gland adenocarcinoma. Proc Natl Acad Sci USA 82: 6497-6501, 1985.

5. Fukushige S, Matsubara K, Yoshida M, et al: Localization of a novel v-erbB-related gene, c-erbB-2, on human chromosome 17 and its amplification in a gastric cancer cell line. Mol Cell Biol 6: 955-958, 1986.

6. Shan LQ, Ma S, Qiu XC, et al: A novel recombinant immunotBid with a furin site effectively suppresses the growth of HER2-positive osteosarcoma cells in vitro. Oncol Rep 25: 325-331, 2011.
7. Scotlandi K, Manara MC, Hattinger CM, et al: Prognostic and therapeutic relevance of HER2 expression in osteosarcoma and Ewing's sarcoma. Eur J Cancer 41: 1349-1361, 2005.

8. Gorlick R, Huvos AG, Heller G, et al: Expression of HER2/ erbB-2 correlates with survival in osteosarcoma. J Clin Oncol 17: 2781-2788, 1999

9. Akatsuka T, Wada T, Kokai Y, et al: ErbB2 expression is correlated with increased survival of patients with osteosarcoma. Cancer 94: 1397-1404, 2002.

10. Qiu XC, Xu YM, Wang F, et al: Single-chain antibody/activated BID chimeric protein effectively suppresses HER2-positive tumor growth. Mol Cancer Ther 7: 1890-1899, 2008.

11. Riedl SJ and Shi Y: Molecular mechanisms of caspase regulation during apoptosis. Nat Rev Mol Cell Biol 5: 897-907, 2004.

12. Slee EA, Adrain C and Martin SJ: Executioner caspase-3, -6, and -7 perform distinct, non-redundant roles during the demolition phase of apoptosis. J Biol Chem 276: 7320-7326, 2001.

13. Ruchaud S, Korfali N, Villa P, et al: Caspase-6 gene disruption reveals a requirement for lamin A cleavage in apoptotic chromatin condensation. EMBO J 21: 1967-1977, 2002.

14. Srinivasula SM, Ahmad M, MacFarlane M, et al: Generation of constitutively active recombinant caspases- 3 and -6 by rearrangement of their subunits. J Biol Chem 273: 10107-10111, 1998.

15. Chen SY, Yang AG, Chen JD, et al: Potent antitumour activity of a new class of tumour-specific killer cells. Nature 385: 78-80, 1997.

16. Batra JK, Kasprzyk PG, Bird RE, Pastan I and King CR: Recombinant anti-erbB2 immunotoxins containing Pseudomonas exotoxin. Proc Natl Acad Sci USA 89: 5867-5871, 1992.

17. Hwang J, Fitzgerald DJ, Adhya S and Pastan I: Functional domains of Pseudomonas exotoxin identified by deletion analysis of the gene expressed in E. coli. Cell 48: 129-136, 1987.

18. Siegall CB, Chaudhary VK, FitzGerald DJ and Pastan I: Functional analysis of domains II, Ib, and III of Pseudomonas exotoxin. J Biol Chem 264: 14256-14261, 1989.

19. Jinno Y, Ogata M, Chaudhary VK, et al: Domain II mutants of Pseudomonas exotoxin deficient in translocation. J Biol Chem 264: 15953-15959, 1989.

20. Siegall CB, Ogata M, Pastan I and FitzGerald DJ: Analysis of sequences in domain II of Pseudomonas exotoxin A which mediate translocation. Biochemistry 30: 7154-7159, 1991.

21. Xu YM, Wang LF, Jia LT, et al: A caspase- 6 and anti-human epidermal growth factor receptor-2 (HER2) antibody chimeric molecule suppresses the growth of HER2-overexpressing tumors. J Immunol 173: 61-67, 2004.

22. Pastan I, Chaudhary V and FitzGerald DJ: Recombinant toxins as novel therapeutic agents. Annu Rev Biochem 61: 331-354, 1992.

23. Springer CJ and Niculescu-Duvaz I: Prodrug-activating systems in suicide gene therapy. J Clin Invest 105: 1161-1167, 2000.

24. Yazawa K, Fisher WE and Brunicardi FC: Current progress in suicide gene therapy for cancer. World J Surg 26: 783-789, 2002.

25. Horowitz J: Adenovirus-mediated p53 gene therapy: overview of preclinical studies and potential clinical applications. Curr Opin Mol Ther 1: 500-509, 1999.

26. Jia LT, Zhang LH, Yu CJ, et al: Specific tumoricidal activity of a secreted proapoptotic protein consisting of HER2 antibody and constitutively active caspase-3. Cancer Res 63: 3257-3262, 2003.

27. Zhao J, Zhang LH, Jia LT, et al: Secreted antibody/granzyme B fusion protein stimulates selective killing of HER2-overexpressing tumor cells. J Biol Chem 279: 21343-21348, 2004.

28. Yu CJ, Jia LT, Meng YL, et al: Selective proapoptotic activity of a secreted recombinant antibody/AIF fusion protein in carcinomas overexpressing HER2. Gene Ther 13: 313-320, 2006. 